\title{
Optical coherence tomography in gynecology: a narrative review
}

Mikhail Kirillin

Tatiana Motovilova

Natalia Shakhova 


\title{
Optical coherence tomography in gynecology: a narrative review
}

\author{
Mikhail Kirillin, ${ }^{a, *}$ Tatiana Motovilova, ${ }^{b}$ and Natalia Shakhova ${ }^{a}$ \\ anstitute of Applied Physics RAS, Nizhny Novgorod, Russia \\ ${ }^{b}$ Nizhny Novgorod State Medical Academy, Nizhny Novgorod, Russia
}

\begin{abstract}
Modern gynecologic practice requires noninvasive diagnostics techniques capable of detecting morphological and functional alterations in tissues of female reproductive organs. Optical coherence tomography (OCT) is a promising tool for providing imaging of biotissues with high resolution at depths up to $2 \mathrm{~mm}$. Design of the customized probes provides wide opportunities for OCT use in gynecology. This paper contains a retrospective insight into the history of OCT employment in gynecology, an overview of the existing gynecologic OCT probes, including those for combination with other diagnostic modalities, and state-of-the-art application of OCT for diagnostics of tumor and nontumor pathologies of female genitalia. Perspectives of OCT both in diagnostics and treatment planning and monitoring in gynecology are overviewed. ๑ The Authors. Published by SPIE under a Creative Commons Attribution 3.0 Unported License. Distribution or reproduction of this work in whole or in part requires full attribution of the original publication, including its DOI. [DOI: 10.1117/1.JBO.22.12.121709]
\end{abstract}

Keywords: optical coherence tomography; gynecology; differential diagnostics; treatment monitoring.

Paper 170604SSVR received Sep. 15, 2017; accepted for publication Nov. 14, 2017; published online Dec. 5, 2017.

\section{Introduction}

Modern clinical practice demonstrates a strong requirement for noninvasive diagnostics techniques capable of providing improved diagnostic efficiency. Among other medical areas, this demand is especially sounding in gynecology, where the problems of untimely diagnostics of neoplasia and latent inflammations affect female reproductive function or lead to cancer development.

While the incidence of invasive cervical cancer has decreased in the last three decades, the rate of cervical dysplasia has significantly increased, ${ }^{1}$ especially in women of reproductive age. ${ }^{2}$ In this respect, diagnosis, management, and follow-up of preinvasive cervical lesions are now a major public health challenge. Combination of colposcopy and directed biopsy is currently an accepted management technique; however, it has low specificity resulting in a fairly large absolute number of overtreatment cases. ${ }^{3}$ Previous studies have shown that unnecessary invasive diagnostic procedures often lead to associated complications, ${ }^{4}$ including adverse birth outcomes. ${ }^{5,6}$

In diagnostics of inflammatory diseases and reproductive disorders, laparoscopy is a standard procedure for examining pelvic pathologies; however, the origin of disorders often cannot be revealed. ${ }^{7}$ Disorders in female fertility are often caused by chronic inflammatory processes within the uterus. ${ }^{8}$ Hysteroscopy, a standard diagnostic tool based on macroscopic examination, reveals chronic endometritis in only $35 \%$ of cases $^{9}$ and should be complemented by an invasive endometrial biopsy. ${ }^{10}$ In this respect, noninvasive diagnostic techniques for gynecology applications are required.

Optical coherence tomography (OCT) is a modern imaging modality capable of providing images of biotissue structure with micron-range spatial resolution ${ }^{11}$ anticipated in the late $1980 \mathrm{~s}^{12}$

*Address all correspondence to: Mikhail Kirillin, E-mail: mkirillin@yandex.ru and introduced in the early 1990s. ${ }^{13}$ Recent achievements in OCT technology provide imaging of biotissues with resolution down to units of microns at depths up to units of mm. After pioneering applications in ophthalmology, OCT was actively introduced into other clinical areas, including gynecology.

Pioneering clinical applications of OCT in gynecology go back to the late 1990s when the first in vivo OCT images of cervix in norm and in course of neoplasia development were obtained. ${ }^{14,15}$ OCT images of pelvic organs (ovaries and fallopian tubes) were obtained in course of standard laparoscopy procedure, and OCT images of endometrium were obtained in course of hysteroscopy. ${ }^{16}$ Furthermore, the OCT studies in gynecology were directed toward revealing the capabilities of OCT in monitoring pathomorphologic and pathophysiologic alterations, which can be used both for diagnostics and treatment monitoring. The first ex vivo study in gynecologic OCT demonstrated the capability of human oviduct imaging of samples after hysterectomy for leiomyomatosis. ${ }^{17}$ An in vitro study ${ }^{18}$ demonstrated that, in OCT images of cervical and uterine tissues in norm and pathology stressing, OCT provides imaging at a resolution higher than any currently available noninvasive clinical imaging technology. Another study on postmortem samples of endometrium and ovary compared OCT images with histology and demonstrated capabilities of OCT in diagnostics of endometritis and ovary neoplasms, and the high potential of laparoscopic OCT applications was emphasized. ${ }^{19}$

Further progress in the introduction of OCT into clinical gynecologic practice aimed at developing customized OCT probes suitable for standard diagnostic procedures and accumulation of OCT image atlases of pelvic organ tissues in norm and pathologies. Recent studies concentrated on application of OCT for nontumor gynecologic pathologies while development of numerical processing and diagnostic image quantification increased diagnostic accuracy against both sole application of standard diagnostic techniques and visual evaluation of diagnostic OCT images. 
The structure of this review is organized as follows: Sec. 2 reviews the technical customization aimed at making OCT technology most compatible with standard diagnostic gynecologic procedures and the combination of OCT with other modern diagnostic modalities. Section 3 overviews the application of OCT for revealing and classification of neoplasia of gynecologic localization, while Sec. 4 discusses the application of OCT for detection of inflammatory diseases and treatment monitoring, paying special attention to application of numerical methods for diagnostic accuracy enhancement. The essential inclusion criterion for this review was reporting either ex vivo or in vivo results on OCT inspection of human female reproductive organs tissue starting from the late 1990s until now.

\section{Customization of Optical Coherence Tomography Probes for Gynecology Applications}

One of the essential aims of OCT customization for gynecologic applications is the construction of the fiber optic-based system equipped with the probe compatible with standard hysteroscopes. While inspection of cervix and vulva does not require special probe configuration and standard laparoscopes employed for fallopian tubes inspection are compatible with most fiber-optic probes, inspection of uterus requires customization, and compatibility of an OCT probe with a hysteroscope is challenging. The first trials on OCT hysteroscopy were performed with a system with a fiber-optics probe with a diameter of $2.7 \mathrm{~mm}$, compatible with a rigid hysteroscope. ${ }^{16}$ The further development of this technology, its enhancement by employing polarization-sensitive OCT imaging, and clinical applications are overviewed in Ref. 20.

In addition to miniaturization, a way to enhance OCT probes consists of the ability for circular and three-dimensional (3-D) scanning; although such systems are usually not compatible with hysteroscopes, they can be efficiently used for OCT colposcopy. A recent paper ${ }^{21}$ reports on an OCT system for colposcopy featuring two probe designs. The first design allows for circular scanning while the other is capable of 3-D imaging over a $4.6 \times 4.6 \mathrm{~mm}^{2}$ area.

A particular issue to be accounted for when using contact fiber-optics probes is tissue compression induced by contact of the probe with tissue surface. The probe pressure to the tissue may induce deformation, affecting the optical properties of tissue and, thus, the OCT image. A special device conjugated with fiber-optics OCT probe was reported in Ref. 22, allowing control of probe pressure in course of OCT colposcopy.

Another approach stimulating development of OCT probes for gynecologic applications is the combination of OCT with other diagnostic modalities, including both standard and perspective ones. Complementary use of OCT imaging and positron detection resulted in the development of a fiber-based probe where the Fourier-domain OCT for volumetric imaging is surrounded by plastic scintillating fiber tips for preferential detection of local positron activity. ${ }^{23}$ The system is aimed at intraoperational detection of ovary cancer.

Another combination method proposed for diagnostics of ovarian cancer includes OCT, ultrasound (US), and photoacoustic imaging (PAI). ${ }^{24}$ The overall diameter of the trimodality probe of the developed system is $5 \mathrm{~mm}$, which is suitable for a standard 5- to 12.5-mm endoscopic/laparoscopic port. The probe combines a ball-lensed OCT sample arm probe, a multimode fiber for PAI, and a high-frequency US transducer with
35-MHz center frequency. Simultaneous employment of PAI allows for blood vessel detection, especially at depths beyond the OCT imaging range.

Modern trends in optical biopsy tend to combine OCT with microscopy imaging. A recent study reports on the development of a combined OCT-microscopy system aimed at the detection of uterine cervix cancer. ${ }^{25}$ The system provides OCT imaging under microscopic guidance and was tested in ex vivo samples obtained by an electrosurgical excision procedure. The approach was demonstrated to be efficient in the detection of high-grade squamous intraepithelial lesions.

A recent study suggests that the fallopian tubes play an important role in the origin of ovarian cancer, making it a critical tissue to timely detect the pathology at an early stage. ${ }^{26}$ It was shown that fluorescence imaging of human ovarian and fallopian tube tissue is promising for early cancer detection. ${ }^{27}$ A combination of fluorescence-based techniques and OCT seems to provide additional benefits. ${ }^{28-30}$ An endoscopic probe for the conjugation of OCT with wide-field falloposcopy ${ }^{31}$, as well as multimodal imaging of ovarian tissue using OCT and second-harmonic generation microscopy, was also reported. ${ }^{32}$

\section{Optical Coherence Tomography Diagnostics of Cancer in Gynecology}

\subsection{Optical Coherence Tomography Diagnostics of Cervix}

The growth of early forms of cervical neoplasia in women of reproductive age requires effective diagnosis. Traditional colposcopy suffers from low specificity that leads to an increasing number of invasive diagnostic procedures and overtreatment. ${ }^{33}$ Thus, there is a strong need for diagnostic techniques, preferably noninvasive, capable of detection cervical neoplasia at early stages with high accuracy. OCT has extremely high potential as an efficient solution for this problem. ${ }^{34}$ The first clinical experience of OCT application for cervix diagnostic was gained in $1997^{16}$ when typical OCT images of cervical tissue in norm and neoplasia were obtained. It was demonstrated that the OCT images of cervix in norm feature stratified squamous cell epithelium and connective tissue stroma with vessels, while a structureless image is an indicator of neoplasia. Typical images of cervix in norm and in cases of nontumor and tumor pathologies are shown in Fig. 1. In 1998 in Ref. 16, the differences in OCT images of cervix in patients with hypoestrogenia and hyperestrogenia were demonstrated, manifested by the thickness and the density of the squamous epithelium. Separately, the images of cervix of unpregnant and pregnant women were compared. Typical images for patients with precancer state [cervical intraepithelial neoplasia (CIN) II] were also reported and qualitative criteria to distinguish precancer alterations were formulated. In a further study, ${ }^{35} 50$ patients with CIN I, II, III, metaplasia/ inflammation, and squamous cell carcinoma were enrolled. Typical images for all cases with higher spatial resolution as compared with a previous paper were obtained. It was demonstrated that OCT allows the separation of invasive cancer from a high-grade preinvasive form that potentially avoids unnecessary biopsies. Another group demonstrated the efficacy of OCT in distinguishing between normal and abnormal (human papillomavirus, CIN I to III) cervical tissue. ${ }^{36}$ Numerical scoring employing the average intensity of the OCT signal from the epithelial layer was developed, demonstrating higher accuracy in premenopausal patients as compared with postmenopausal. 

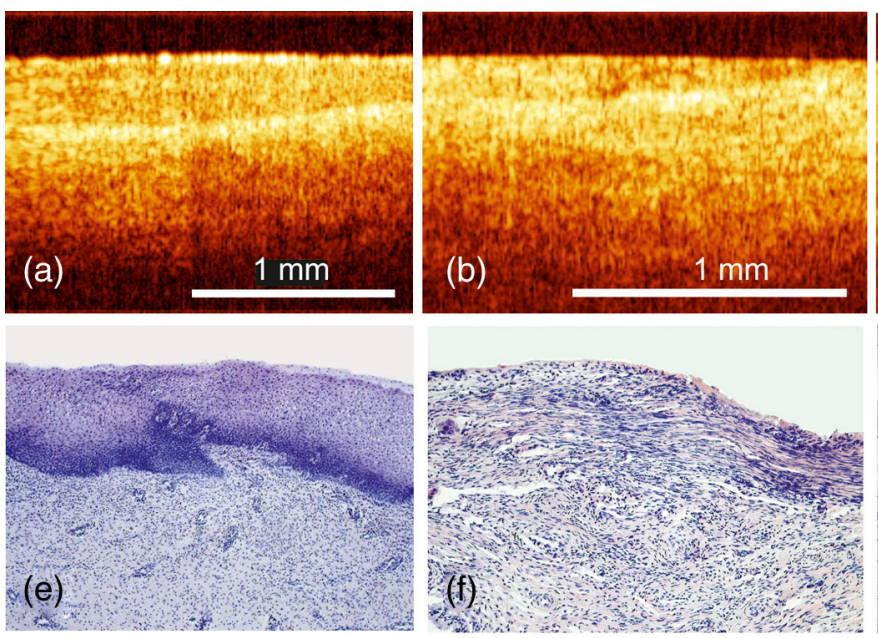
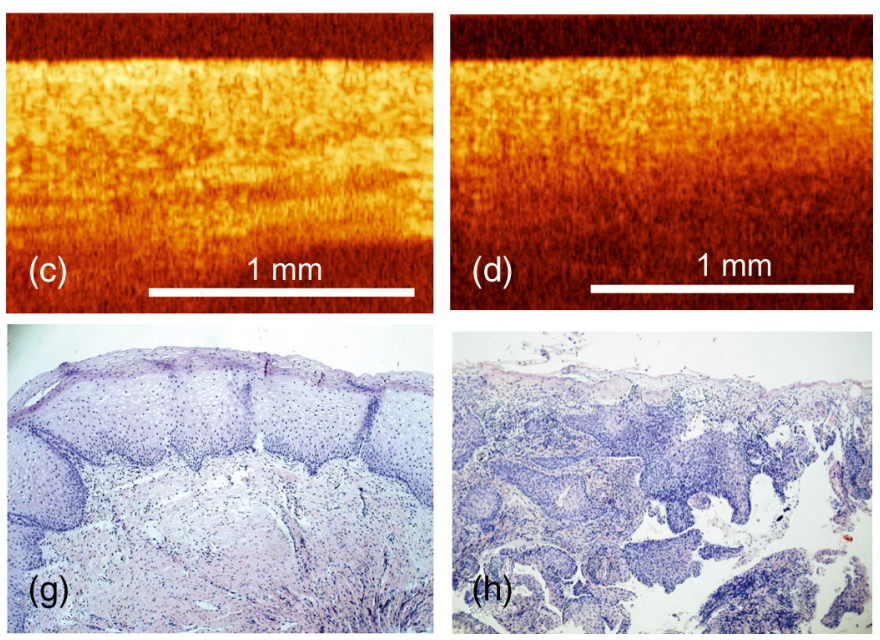

Fig. 1 Typical OCT images and corresponding histology of human cervix: (a, e) norm, (b, f) inflammation (cervicitis), (c, g) CIN I, and (d, h) carcinoma.

As the next step, ${ }^{37}$ the sensitivity and specificity of OCT in the detection of cervical cancer in combination with traditional approaches, visual inspection with acetic acid (VIA) and colposcopy, were determined. Typical images of ectocervix for norm, chronic inflammation, carcinoma in situ, and CIN III were demonstrated. It was shown that OCT provides an increase in specificity in the detection of CIN II from $34 \%$ to $61 \%$ when used in combination with VIA as compared with VIA alone while sensitivity drops from $76 \%$ to $53 \%$. Additional employment of colposcopy results in sensitivity of $46 \%$ and specificity of $69 \%$. Similar evaluation of OCT efficacy alone in detecting cervical neoplasia was carried out later by another group ${ }^{38}$ reporting sensitivity values of $95 \%$ and $96 \%$ and specificity values of $46 \%$ and $29 \%$ for two independent investigators, while interobserver agreement kappa equal to 0.68 is reported. The continuation of this work ${ }^{39}$ reports high-resolution images of healthy cervical tissue, CIN I, II, and III together with corresponding histology images. Reported values for sensitivity are $98 \%$ and $96 \%$ for two investigators with specificity of $39 \%$ and $41 \%$, respectively, when the threshold for tumor state is set at CIN I. Shifting the threshold to CIN II results in sensitivity of $86 \%$ and $84 \%$ while specificity dramatically rises to $64 \%$ and $60 \%$, respectively. Similar to previous study, interobserver agreement kappa is reported as 0.69 and 0.62 for threshold of CIN I and CIN II, respectively.

The idea of combination of OCT inspection with VIA proposed in Ref. 37 gains its continuation in another prospective study with 1000 patients enrolled. ${ }^{40}$ When the threshold was chosen as CIN II, the sensitivity and specificity of VIA only in detecting lesions were $43 \%$ and $96 \%$, respectively, while in combination OCT the sensitivity increased to $62 \%$ with a specificity of $80 \%$. The authors stress that introduction of the OCT image numerical processing could provide additional benefit to the technology. Similar to Ref. 37, the next step was to determine possible benefits for combination of OCT and traditional colposcopy. ${ }^{41}$ The study included 299 patients and demonstrated that the sensitivity for CIN II or higher decreased by adding OCT to colposcopy, but the specificity increased from $83 \%$ to $93 \%$. Inspection of loop electrosurgical excision procedure specimens with a system conjugating OCT with microscopy for detection of high-grade cervical dysplasia ( $\geq$ CIN II) allowed achieving sensitivity of $88 \%$ and $84 \%$ and specificity of $69 \%$ and $65 \%$ for two independent investigators, respectively, with an agreement coefficient kappa of $0.85 .^{25}$

Thus, the overviewed studies demonstrate the perspectives of OCT in diagnostics of cervical neoplasia; however, further improvement of the technique and development of diagnostic protocols are required.

\subsection{Optical Coherence Tomography Diagnostics of Vulva}

Recent years are characterized by the increase in vulvar intraepithelial neoplasia (VIN) occurrences requiring improved diagnostic techniques. ${ }^{42}$ Although cervical tissue is mucosa, while vulvar tissue is skin, due to similar morphologic structure of cervical tissue and vulvar tissue represented by an epidermal layer with an underlying connective tissue layer, the approaches for employment OCT in evaluation of vulva pathologies are similar to that employed for cervix. In the pioneering paper in OCT inspection of vulva, ${ }^{35}$ the OCT images of vulva with Paget's disease were reported and compared with corresponding histology images. In later studies, ${ }^{43,44}$ it was demonstrated that OCT is efficient in differentiating between normal and VIN tissue. Two criteria were employed for differentiation: the thickness of the epidermal layer measured from an OCT image and the attenuation coefficient of this layer extracted from in-depth OCT-signal attenuation. Epidermis thickness was found as $0.19 \pm 0.04 \mathrm{~mm}$ in normal vulvar tissue and $0.56 \pm 0.22$ for VIN. The attenuation coefficient amounted to $2.1 \pm 1.4 \mathrm{~mm}^{-1}$ and $6.2 \pm 2.1 \mathrm{~mm}^{-1}$ for norm and VIN, respectively. Furthermore, these criteria were extended to four classes of OCT images of vulva: normal, benign, VIN, and vulvar squamous cell carcinoma (VSCC) ${ }^{45}$ It was demonstrated that use of epidermal thickness ensures distinguishing between normal/ benign tissue and VIN/VSCC tissue with sensitivity of $100 \%$ and specificity of $80 \%$. Employment of the attenuation coefficient provides sensitivity of $100 \%$ and specificity of $70 \%$. OCT was demonstrated to be efficient in the detection of margins of VSCC, which is important for determining the stage of the disease. 


\subsection{Optical Coherence Tomography Diagnostics of Ovary}

Ovarian cancer ranks fifth in cancer deaths among women, accounting for more deaths than any other cancer of the female reproductive system. A woman's risk of getting ovarian cancer during her lifetime is about 1 in 75 , and a lifetime chance of dying from ovarian cancer is about 1 in $100 .^{46}$ Survival is strongly associated with the stage of cancer at diagnosis; however, currently, there exist no efficient approaches for early detection and screening of ovarian cancer, ${ }^{47}$ and further research to identify opportunities to solve this problem is needed. ${ }^{48}$ One of potential approaches consists of complementary use of modern imaging techniques, including OCT.

For the first time, OCT images of ovarian tissue with cystadenocarcinoma and adenoma together with corresponding histology images were reported in $1999 ;{ }^{19}$ the authors stressed a high potential of laparoscopic applications of OCT. A laparoscopic OCT device was employed for diagnostics of ovarian cancer in paper. ${ }^{49}$ The paper features OCT images of postmenopausal ovary in norm, endometriosis, serous cystadenoma, and endometrioid adenocarcinoma with corresponding histology images. Typical features of these OCT images were formulated. A later study demonstrated the potential of combining OCT with PAI and US in vivo ${ }^{24}$ and positron detection ex vivo ${ }^{50}$ in revealing malignant areas in the ovary.

In further investigations, ${ }^{51,52}$ the benefits from numerical processing of OCT images of ovary ex vivo were demonstrated for the obtained image set. The first study ${ }^{52}$ proposed employment of the use of scattering coefficient of the ovary tissue derived from an OCT image as a criterion to separate normal tissue from a malignant one. The coefficient is extracted basing on the confocal point spread function of the OCT system and Beer's law. Reported values for normal and malignant groups are $2.41 \pm 0.59 \mathrm{~mm}^{-1}$ and $1.55 \pm 0.46 \mathrm{~mm}^{-1}$, respectively. The approach distinguished normal ovary from a malignant one with sensitivity from $80 \%$ to $61 \%$ and specificity from $75 \%$ to $90 \%$ when the threshold value varied from 2 to $1.67 \mathrm{~mm}^{-1}$. The second study ${ }^{51}$ analyzed angle-resolved scattering properties derived from OCT images when the scattering coefficient was derived for different directions of an A-scan, and the mean correlation coefficient for these values was calculated. It was shown that this coefficient is higher for normal tissue than for a malignant one, providing $100 \%$ specificity and sensitivity for the considered set of OCT images from 10 ex vivo ovaries. Additional benefits in detection malignancy in the ovary are provided by polarization-sensitive OCT modality providing both conventional OCT image and conjugated phase retardation map. ${ }^{53,54}$ Phase retardation map reflects the birefringence properties of the tissue, thus allowing evaluation of the collagen state. Disrupted collagen is usually an indicator of a malignancy presence. Average phase retardation value derived from phase retardation maps was employed as another criterion in line with scattering coefficient to separate norm from malignancy, providing sensitivity of $43 \%$ and specificity of $100 \%$. Being used in combination with scattering coefficient, it ensured both specificity and sensitivity of $100 \%$. An overview of employment of OCT for characterization of ovarian tissue was given in a review paper. ${ }^{55}$ Recent animal studies aimed at proving the efficacy of OCT in ovary transplantation ${ }^{56,57} \mathrm{dem}$ onstrated high potential of OCT in detecting metastases in the ovary, including micrometastases. ${ }^{56} \mathrm{~A}$ recent $e x$ vivo study employing full-field $\mathrm{OCT}^{58,59}$ demonstrated that application of standard image characterization (calculation of mean, variance, skewness, kurtosis, and entropy) to OCT images of ovaries allowed to differentiate normal ovary from a malignant one with a sensitivity of $92 \%$ and specificity of $88 \%$. It is worth mentioning that, despite quite extensive studies on OCT applications for detection of ovarian cancer, most of the works are ex vivo, and the technology is yet to be translated to clinical practice. The combination of OCT with a falloscope by a custom-designed probe tested in animal study ${ }^{31}$ is a significant step toward clinical application.

\section{Optical Coherence Tomography Diagnostics of Nontumor Pathologies and Treatment Monitoring In Gynecology}

Although the main role of OCT in gynecology as an in vivo technique of morphological inspection is the detection of cancer, it can serve as a tool for the detection and classification of other pathologies of gynecologic localizations, namely inflammatory diseases and their manifestation, such as edema, sclerosis, and others. The localizations include uterine cavity (endometrium pathologies), cervix, vulva, and fallopian tubes.

One of the pioneering papers on OCT in gynecology ${ }^{19}$ reported typical images of normal peritoneal surface of uterus and endometriosis with corresponding histology images. The ex vivo OCT images of normal peritoneal surface feature adipose tissue and underlying uterine myometrium while endometriosis was manifested by appearance of characteristic glandular structures. Later, OCT images of endometrioid foci were reported for in vivo studies. ${ }^{60}$

Further laparoscopic OCT was used to diagnose fallopian tubes in cases of silent inflammation and infertility of unknown origin. OCT images of fallopian tubes with latent inflammation were obtained, and criteria for detection of chronic salpingitis with OCT were developed. ${ }^{22,61,62}$ A numerical approach for OCT image analysis aimed at scoring the feature of an OCT image typical for manifestations of sclerosis and edema was developed. Sclerosis is usually manifested by higher OCT-signal level associated with higher scattering while edema is manifested by well-shaped darker areas associated with presence of extracellular liquid. It was demonstrated that employment of OCT in addition to standard laparoscopy only increased sensitivity from $44 \%$ to $90 \%$ and specificity from $67 \%$ to $81 \%$, while numerical scoring of OCT images achieved sensitivity and specificity of $100 \%$ and $96 \%$, respectively, for a set of 61 OCT images. Typical images of fallopian tubes in norm and in cases of inflammation are shown in Fig. 2.

Recent studies report on the application of OCT for detection and classification of endometritis. ${ }^{62,63}$ Numerical processing of diagnostic OCT images allowed the development of a scoring system to quantify endometritis grade. The system is based on evaluation of the grade of sclerosis, which is a constant morphologic indicator of chronic endometritis.

OCT studies of cervix also demonstrate that OCT can be a tool to evaluate the hormonal state of cervix ${ }^{16,36}$ reporting on differences in images for pre- and postmenopausal patients. The OCT technique also demonstrated to be efficient in revealing nontumor cervix alterations in combination with a mechanical compression approach when typical layered structure is not observed. ${ }^{22}$ Evaluation of a diagnostic OCT image of suspicious cervix region after inspection with controlled mechanical compression of an OCT probe to cervical tissue avoided unnecessary biopsies in particular cases. 

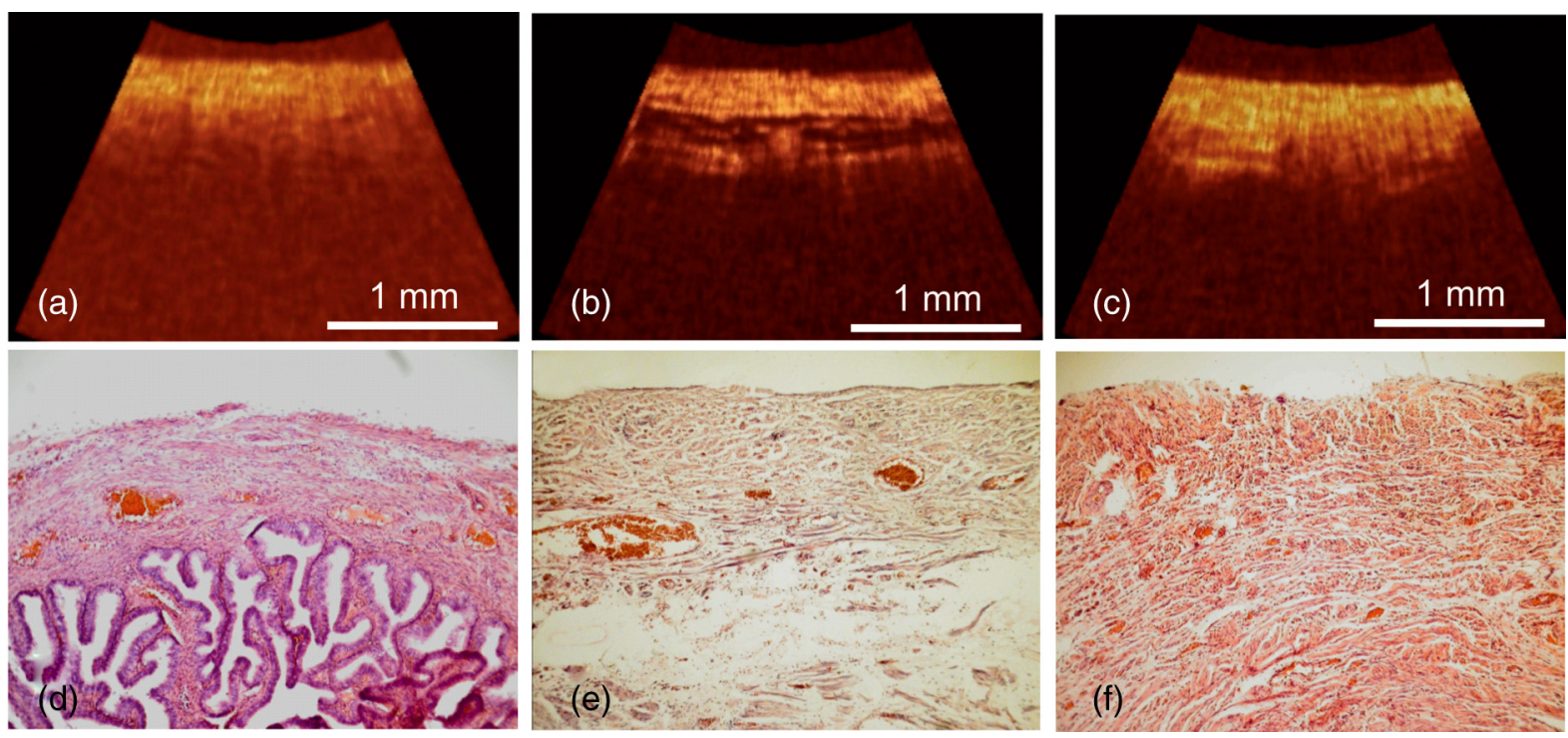

Fig. 2 Typical OCT images and corresponding histology of fallopian tube tissue: (a, d) norm, (b, e) inflammation with prevalence of edema, and $(c, f)$ chronic alterations with fibrosis. ${ }^{61}$

OCT was also employed for evaluation of vaginal epithelial damage and monitoring of its treatment with nonoxynol-9 vaginal gel. ${ }^{64}$ Colposcopy was used simultaneously as a standard approach. Epithelial thickness and integrity ${ }^{65}$ were used as scores derived from OCT images to evaluate the state of the tissue. OCT revealed epithelial disruption and thinning not identified by colposcopy.

Animal studies demonstrated that high-resolution OCT can be used noninvasively to evaluate vaginal morphologic features, including epithelial thickness, to assess this protective barrier in transmission of sexually transmitted infections and to monitor tissue response to topical medications and hormonal fluctuations. ${ }^{66-68}$ In clinical studies, ${ }^{69}$ it was shown that cross-polarization OCT can be used for noninvasive monitoring of responses of tumors and adjacent normal tissues to photodynamic therapy (PDT) of cervical cancer, thus, being useful for evaluation of the effectiveness of therapy and for choosing optimal treatment tactics.

The potential of OCT in employment for screening programs in microbicenosis studies was also demonstrated. ${ }^{70}$

\section{Conclusion}

Optical techniques are used for gynecologic applications due to their noninvasiveness and high diagnostic accuracy compared with traditional diagnostic techniques. ${ }^{34}$ OCT has a special potential in gynecologic applications due to high-resolution imaging, customized probes, and sensitivity to both morphologic and functional changes. ${ }^{6,71,72}$ The main advantages of applications of OCT technology in gynecology are noninvasiveness and ease of operation; generally, its usage is similar to that of US technology.

Employment of customized OCT probes, especially in combination with other diagnostic modalities, provide additional diagnostic information to avoid unnecessary biopsies. In diagnostics of tumor pathologies, OCT not only reveals the pathology to detect the stage of the disease and to plan the treatment. In particular cases, extremely high values for specificity and sensitivity are demonstrated with OCT while traditional techniques provide comparatively low diagnostic accuracy. Employment of OCT was demonstrated to be efficient in diagnostics of latent forms of inflammation, which is important in diagnostics of reproductive disorders in females.

The main drawbacks of the OCT employment in gynecology consist of high requirements of the OCT probes-in diagnostics of cervix and vulva, sterilization of the probes is critical; in diagnostics of ovaries and fallopian tubes, the probe geometry should fit a standard laparoscope; and in diagnostics of endometrium, it should fit a standard hysteroscope. Another OCT drawback is the requirement for correct interpretation of the obtained diagnostic OCT image, and a clinician who is not familiar with the technique may fail to provide a correct diagnosis. In this respect, numerical image processing and analysis are of importance, as they were demonstrated to increase the diagnostic accuracy of OCT in a number of gynecologic applications. An associated drawback, which follows from OCT studies of cervix being the most extensive investigation from all OCT gynecologic applications, is comparatively low specificity of OCT. Potential ways for increasing the specificity of OCT include application of image enhancement approaches, such as optical clearing ${ }^{73}$ or mechanical compression. Table 1 summarizes reported studies on OCT gynecologic applications, advantages, and drawbacks for every particular application and achieved sensitivity and specificity, where applicable.

The overviewed OCT applications in gynecology indicate high potential of this technique in treatment monitoring; however, no such gynecologic applications were reported so far. Recently, OCT was reported as an efficient tool for treatment monitoring of $\operatorname{skin}^{74-76}$ and mucous tissues ${ }^{62,77}$ of other localizations. One of the modern trends in gynecology is the use of organ-preserving treatment approaches, in particular, PDT. ${ }^{78-}$ ${ }^{80}$ Demonstrated capabilities of OCT in the detection of morphological alterations can be employed for monitoring PDT of gynecologic localizations for treatment optimization and personalization. Additional advantages in diagnostic accuracy can be provided by advanced OCT modalities, such as polarization-sensitive OCT, providing information about birefringent 
Table 1 Application of OCT for diagnostics in clinical gynecology: an overview.

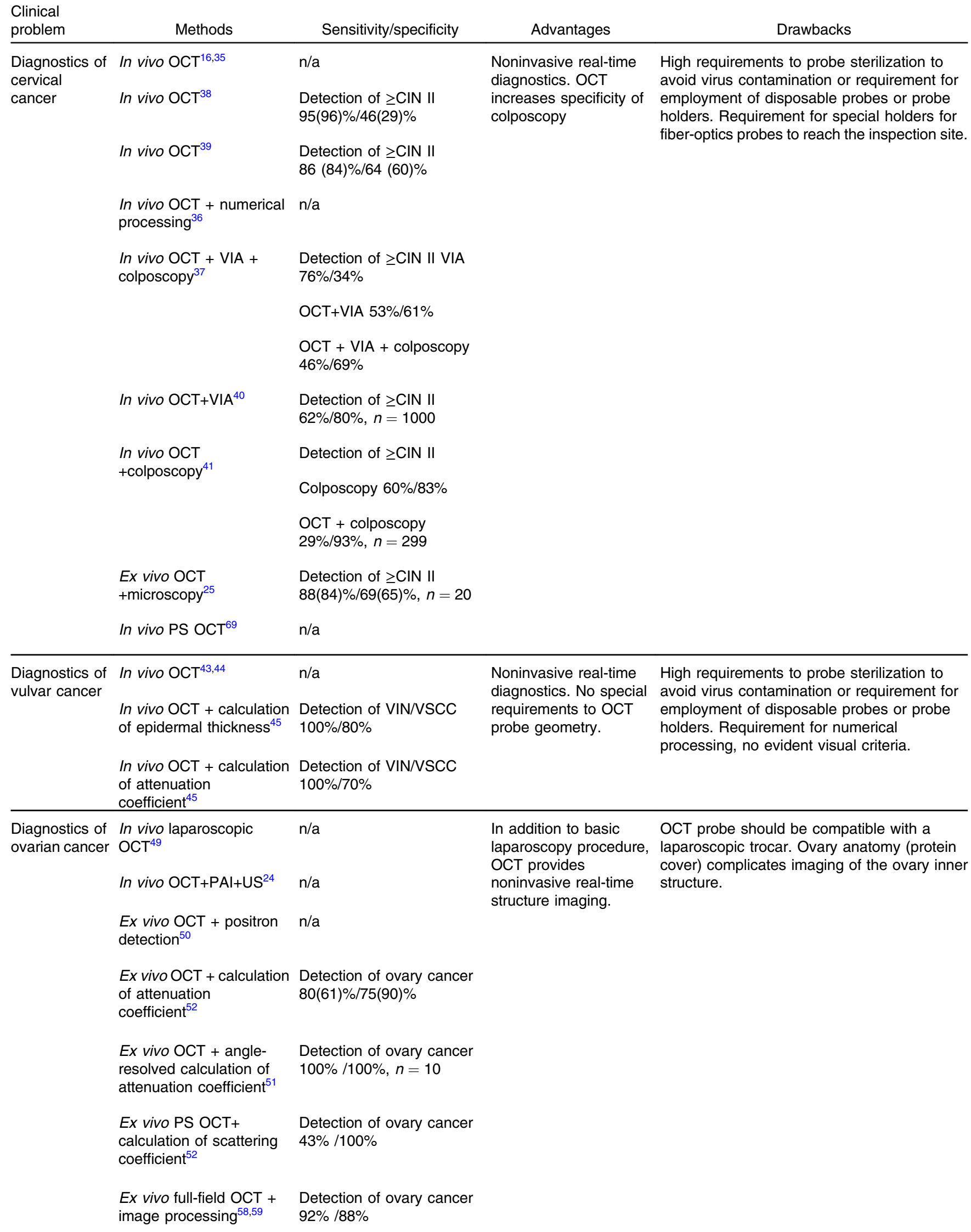


Table 1 (Continued).

\begin{tabular}{|c|c|c|c|c|}
\hline $\begin{array}{l}\text { Clinical } \\
\text { problem }\end{array}$ & Methods & Sensitivity/specificity & Advantages & Drawbacks \\
\hline $\begin{array}{l}\text { Diagnostics of } \\
\text { endometriosis }\end{array}$ & $\begin{array}{l}\text { Ex vivo } \mathrm{OCT}^{19} \\
\text { In vivo } \mathrm{OCT}^{60}\end{array}$ & $\mathrm{n} / \mathrm{a}$ & $\begin{array}{l}\text { Noninvasive real-time } \\
\text { diagnostics. High } \\
\text { potential in differential } \\
\text { diagnostics of small } \\
\text { types of endometriosis. }\end{array}$ & $\begin{array}{l}\text { OCT probe should be compatible with a } \\
\text { laparoscopic trocar. Too little observations } \\
\text { reported. }\end{array}$ \\
\hline $\begin{array}{l}\text { Diagnostics of } \\
\text { fallopian tubes } \\
\text { disorders }\end{array}$ & $\begin{array}{l}\text { In vivo OCT + } \\
\text { laparoscopy + numerical } \\
\text { processing }^{61}\end{array}$ & $\begin{array}{l}\text { Detection of salpingitis } \\
\text { Laparoscopy } 44 \% / 67 \% \\
\text { OCT + laparoscopy } \\
90 \% / 81 \% \\
\text { OCT + laparoscopy + } \\
\text { numerical processing } \\
100 \% / 96 \%, n=61\end{array}$ & $\begin{array}{l}\text { Noninvasive real-time } \\
\text { diagnostics. Unique } \\
\text { ability to obtain in vivo } \\
\text { information on structure } \\
\text { of muscular layer of a } \\
\text { fallopian tube. } \\
\text { Additional information } \\
\text { for differential } \\
\text { diagnostics of } \\
\text { morphological forms of } \\
\text { inflammatory diseases. }\end{array}$ & $\begin{array}{l}\text { OCT probe should be compatible with a } \\
\text { laparoscopic trocar. }\end{array}$ \\
\hline $\begin{array}{l}\text { Diagnostics of } \\
\text { endometritis }\end{array}$ & $\begin{array}{l}\text { In vivo OCT + numerical } \\
\text { processing }^{62,63}\end{array}$ & $\mathrm{n} / \mathrm{a}$ & $\begin{array}{l}\text { Noninvasive real-time } \\
\text { diagnostics. Additional } \\
\text { information for } \\
\text { differential diagnostics } \\
\text { of morphological forms } \\
\text { of inflammatory } \\
\text { diseases. }\end{array}$ & $\begin{array}{l}\text { OCT probe should be compatible with a } \\
\text { hysteroscope, incompatibility with office-based } \\
\text { hysteroscope. }\end{array}$ \\
\hline $\begin{array}{l}\text { Diagnostics of } \\
\text { nontumor } \\
\text { cervix } \\
\text { alterations }\end{array}$ & In vivo $\mathrm{OCT}^{16,22,36}$ & $\mathrm{n} / \mathrm{a}$ & $\begin{array}{l}\text { Noninvasive real-time } \\
\text { diagnostics. Additional } \\
\text { information for } \\
\text { differential diagnostics } \\
\text { of morphological forms } \\
\text { of inflammatory } \\
\text { diseases. }\end{array}$ & $\begin{array}{l}\text { High requirements to probe sterilization to } \\
\text { avoid virus contamination or requirement for } \\
\text { employment of disposable probes or probe } \\
\text { holders. Requirement for special holders for } \\
\text { fiber-optics probes to reach the inspection site. }\end{array}$ \\
\hline $\begin{array}{l}\text { Diagnostics of } \\
\text { vaginal } \\
\text { epithelial } \\
\text { damage }\end{array}$ & $\begin{array}{l}\text { In vivo OCT }+ \\
\text { vulvoscopy }^{64,65}\end{array}$ & $\mathrm{n} / \mathrm{a}$ & $\begin{array}{l}\text { Noninvasive real-time } \\
\text { diagnostics. No special } \\
\text { requirements to OCT } \\
\text { probe design. Additional } \\
\text { information for } \\
\text { differential diagnostics } \\
\text { of morphological forms } \\
\text { of inflammatory } \\
\text { diseases. }\end{array}$ & $\begin{array}{l}\text { High requirements to probe sterilization to } \\
\text { avoid virus contamination or requirement for } \\
\text { employment of disposable probes or probe } \\
\text { holders. }\end{array}$ \\
\hline
\end{tabular}

Note: OCT, optical coherence tomography; PS OCT, polarization-sensitive OCT; VIA, visual inspection with acetic acid; CIN, cervical intraepithelial neoplasia; VIN, vulvar intraepithelial neoplasia; VSCC, vulvar squamous cell carcinoma; PAI, photoacoustic imaging; and US, ultrasound. In the brackets, alternative reported value is shown.

properties of tissues and OCT-angiography allowing for highresolution mapping of microcirculation within tissues.

\section{Disclosures}

The authors have nothing to disclose.

\section{Acknowledgments}

The work was supported by the Russian Science Foundation (Project No. 17-15-01264).

\section{References}

1. H. W. Jones and J. A. Rock, Eds., Te Linde's Operative Gynecology, 11th ed., Wolters Kluwer, Philadelphia (2015).

2. M. Bazaz et al., "Cervical cancer literacy in women of reproductive age and its related factors," J. Cancer Educ. 1-8 (2017).
3. R. A. Mustafa et al., "Systematic reviews and meta-analyses of the accuracy of HPV tests, visual inspection with acetic acid, cytology, and colposcopy," Int. J. Gynecol. Obstet. 132, 259-265 (2016).

4. V. A. Moyer, "Screening for cervical cancer: US preventive services task force recommendation statement," Ann. Intern. Med. 156, 880891 (2012).

5. D. Saslow et al., "American Cancer Society, American Society for Colposcopy and Cervical Pathology, and American Society for Clinical Pathology screening guidelines for the prevention and early detection of cervical cancer," CA: Cancer J. Clin. 62, 147-172 (2012).

6. F. Bruinsma and M. Quinn, "The risk of preterm birth following treatment for precancerous changes in the cervix: a systematic review and metaanalysis," BJOG: Int. J. Obstet. Gynaecol. 118, 1031-1041 (2011).

7. R. Togni, C. L. Benetti-Pinto, and D. A. Yela, "The role of diagnostic laparoscopy in gynecology," Sao Paulo Med. J. 134, 70-73 (2016).

8. K. Kitaya et al., "Chronic endometritis: potential cause of infertility and obstetric and neonatal complications," Am. J. Reprod. Immunol. 75, 13-22 (2016). 
9. H. J. Park et al., "Chronic endometritis and infertility," Clin. Exp. Reprod. Med. 43, 185-192 (2016).

10. P.-E. Bouet et al., "Chronic endometritis in women with recurrent pregnancy loss and recurrent implantation failure: prevalence and role of office hysteroscopy and immunohistochemistry in diagnosis," Fertil. Steril. 105, 106-110 (2016).

11. B. E. Bouma and G. J. Tearney, Handbook of Optical Coherence Tomography, Marcel Dekker, New York (2002).

12. A. Fercher, K. Mengedoht, and W. Werner, "Eye-length measurement by interferometry with partially coherent light," Opt. Lett. 13, 186-188 (1988).

13. D. Huang et al., "Optical coherence tomography," Science 254, 11781181 (1991).

14. A. M. Sergeev et al., "In vivo endoscopic OCT imaging of precancer and cancer states of human mucosa," Opt. Express 1, 432-440 (1997).

15. F. I. Feldchtein et al., "Endoscopic applications of optical coherence tomography," Opt. Express 3, 257-270 (1998).

16. N. M. Shakhova et al., "Endoscopic OCT for imaging of uterine body and cervix pathologies," Proc. SPIE 3251, 59-66 (1998).

17. J. M. Herrmann et al., "Two- and three-dimensional high-resolution imaging of the human oviduct with optical coherence tomography," Fertil. Steril. 70, 155-158 (1998).

18. C. Pitris et al., "High-resolution imaging of gynecologic neoplasms using optical coherence tomography," Obstet. Gynecol. 93, 135-139 (1999).

19. S. Boppart et al., "High resolution imaging of endometriosis and ovarian carcinoma with optical coherence tomography: feasibility for laparoscopic-based imaging," BJOG: Int. J. Obstet. Gynaecol. 106, 10711077 (1999).

20. V. Gelikonov et al., "Fiber-based OCT: from optical design to clinical applications," Chapter 16 in Handbook of Photonics for Biomedical Science, V. V. Tuchin, Ed., pp. 423-444, CRC Press, Boca Raton, London, New York, Washington (2010).

21. L. Duan et al., "Colposcopic imaging using visible-light optical coherence tomography," J. Biomed. Opt. 22, 056003 (2017).

22. M. Kirillin et al., "Towards increase of diagnostic efficacy in gynecologic OCT," Proc. SPIE 8802, 880205 (2013).

23. J. Gamelin et al., "A prototype hybrid intraoperative probe for ovarian cancer detection," Opt. Express 17, 7245-7258 (2009).

24. Y. Yang et al., "Integrated optical coherence tomography, ultrasound and photoacoustic imaging for ovarian tissue characterization," Biomed. Opt. Express 2, 2551-2561 (2011).

25. J. Gallwas et al., "Detection of cervical intraepithelial neoplasia by using optical coherence tomography in combination with microscopy," J. Biomed. Opt. 22, 016013 (2017).

26. R. S. Tuma, "Origin of ovarian cancer may have implications for screening," J. Natl. Cancer Inst. 102(1), 11-13 (2010).

27. T. H. Tate et al., "Multispectral fluorescence imaging of human ovarian and fallopian tube tissue for early-stage cancer detection," J. Biomed. Opt. 21, 056005 (2016).

28. R. A. Wall and J. K. Barton, "Fluorescence-based surface magnifying chromoendoscopy and optical coherence tomography endoscope," J. Biomed. Opt. 17, 086003 (2012).

29. T. Tate et al., "Optical design of an optical coherence tomography and multispectral fluorescence imaging endoscope to detect early stage ovarian cancer," in Int. Optical Design Conf., OSA Technical Digest (2014).

30. M. Keenan et al., "Optical coherence tomography and multispectral fluorescence imaging falloposcope," in Frontiers in Optics, OSA Technical Digest (2014).

31. M. Keenan et al., "Design and characterization of a combined OCT and wide field imaging falloposcope for ovarian cancer detection," Biomed. Opt. Express 8, 124-136 (2017).

32. W. A. Welge et al., "Diagnostic potential of multimodal imaging of ovarian tissue using optical coherence tomography and secondharmonic generation microscopy," J. Med. Imaging 1, 025501 (2014).

33. M. U. Barut et al., "Analysis of sensitivity, specificity, and positive and negative predictive values of smear and colposcopy in diagnosis of premalignant and malignant cervical lesions," Medical Sci. Monitor: Int. Med. J. Exp. Clin. Res. 21, 3860-3867 (2015).

34. T. Novikova, "Optical techniques for cervical neoplasia detection," Beilstein J. Nanotechnol. 8, 1844-1862 (2017).
35. P. Escobar et al., "Diagnostic efficacy of optical coherence tomography in the management of preinvasive and invasive cancer of uterine cervix and vulva," Int. J. Gynecol. Cancer 14, 470-474 (2004).

36. A. F. Zuluaga et al., "Optical coherence tomography: a pilot study of a new imaging technique for noninvasive examination of cervical tissue," Am. J. Obstet. Gynecol. 193, 83-88 (2005).

37. P. Escobar et al., "Optical coherence tomography as a diagnostic aid to visual inspection and colposcopy for preinvasive and invasive cancer of the uterine cervix," Int. J. Gynecol. Cancer 16, 1815-1822 (2006).

38. J. Gallwas et al., "Optical coherence tomography as a non-invasive imaging technique for preinvasive and invasive neoplasia of the uterine cervix," Ultrasound Obstet. Gynecol. 36, 624-629 (2010).

39. J. K. Gallwas et al., "Optical coherence tomography for the diagnosis of cervical intraepithelial neoplasia," Lasers Surg. Med. 43, 206-212 (2011).

40. N. Wulan et al., "Study of the diagnostic efficacy of real-time optical coherence tomography as an adjunct to unaided visual inspection with acetic acid for the diagnosis of preinvasive and invasive neoplasia of the uterine cervix," Int. J. Gynecol. Cancer 20, 422-427 (2010).

41. Z. Liu et al., "Diagnostic efficacy of real-time optical coherence tomography in the management of preinvasive and invasive neoplasia of the uterine cervix," Int. J. Gynecol. Cancer 20, 283-287 (2010).

42. L. N. Hoang et al., "Squamous precursor lesions of the vulva: current classification and diagnostic challenges," Pathology 48, 291-302 (2016).

43. R. Wessels et al., "Optical coherence tomography in vulvar intraepithelial neoplasia," J. Biomed. Opt. 17, 116022 (2012).

44. R. Wessels et al., "Optical biopsy of epithelial cancers by optical coherence tomography (OCT)," Lasers Med. Sci. 29, 1297-1305 (2014).

45. R. Wessels et al., "The value of optical coherence tomography in determining surgical margins in squamous cell carcinoma of the vulva: a singlecenter prospective study," Int. J. Gynecol. Cancer 25, 112-118 (2015).

46. American Cancer Society, Cancer Facts \& Figures 2017, American Cancer Society, Atlanta (2017).

47. B. Kearns et al., "Cost-effectiveness of screening for ovarian cancer amongst postmenopausal women: a model-based economic evaluation," BMC Med. 14, 200 (2016).

48. C. Corzo et al., "Role of fallopian tubes in the development of ovarian cancer," J. Minimally Invasive Gynecol. 24, 230-234 (2017).

49. L. P. Hariri et al., "Laparoscopic optical coherence tomography imaging of human ovarian cancer," Gynecol. Oncol. 114, 188-194 (2009).

50. Y. Yang et al., "Potential role of a hybrid intraoperative probe based on $\mathrm{OCT}$ and positron detection for ovarian cancer detection and characterization," Biomed. Opt. Express 2, 1918-1930 (2011).

51. Y. Yang et al., "Quantitative analysis of angle-resolved scattering properties of ovarian tissue using optical coherence tomography," J. Biomed. Opt. 17, 090503 (2012).

52. Y. Yang et al., "Optical scattering coefficient estimated by optical coherence tomography correlates with collagen content in ovarian tissue," J. Biomed. Opt. 16, 090504 (2011).

53. Y. Yang et al., "Quantitative analysis of estimated scattering coefficient and phase retardation for ovarian tissue characterization," Biomed. Opt. Express 3, 1548-1556 (2012).

54. T. Wang, Y. Yang, and Q. Zhu, "A three-parameter logistic model to characterize ovarian tissue using polarization-sensitive optical coherence tomography," Biomed. Opt. Express 4, 772-777 (2013).

55. T. Wang, M. Brewer, and Q. Zhu, "An overview of optical coherence tomography for ovarian tissue imaging and characterization," Wiley Interdiscip. Rev. Nanomed. Nanobiotechnol. 7, 1-16 (2015).

56. I. T. Peters et al., "Noninvasive detection of metastases and follicle density in ovarian tissue using full-field optical coherence tomography," Clin. Cancer Res. 22, 5506-5513 (2016).

57. S. Takae et al., "Accuracy and safety verification of ovarian reserve assessment technique for ovarian tissue transplantation using optical coherence tomography in mice ovary," Sci. Rep. 7, 43550 (2017).

58. S. Nandy, M. Sanders, and Q. Zhu, "Classification and analysis of human ovarian tissue using full field optical coherence tomography," Biomed. Opt. Express 7, 5182-5187 (2016).

59. S. Nandy, M. Sanders, and Q. Zhu, "Classification of human ovarian tissue using full field optical coherence tomography," Proc. SPIE 10053, 100533D (2017).

60. O. Panteleeva et al., "OCT in difficult diagnostic cases in gynecology," Proc. SPIE 8091, 809124 (2011). 
61. M. Kirillin et al., "Criteria for pathology recognition in optical coherence tomography of fallopian tubes," J. Biomed. Opt. 17, 081413 (2012).

62. M. Kirillin et al., "Quantitative optical diagnostics in pathology recognition and monitoring of tissue reaction to PDT," Proc. SPIE 10417, 104170C (2017).

63. I. A. Kuznetsova et al., "OCT in gynecology," in Optical Coherence Tomography: Technology And Applications, W. Drexler and J. Fujimoto, Eds., pp. 2305-2334, Springer, New York (2015).

64. K. L. Vincent et al., "Optical coherence tomography compared with colposcopy for assessment of vaginal epithelial damage: a randomized controlled trial," Obstet. Gynecol. 118, 1354-1361 (2011).

65. S. Chitchian et al., "Automated segmentation algorithm for detection of changes in vaginal epithelial morphology using optical coherence tomography," J. Biomed. Opt. 17, 116004 (2012).

66. K. L. Vincent et al., "Application of optical coherence tomography for monitoring changes in cervicovaginal epithelial morphology in macaques: potential for assessment of microbicide safety," Sex. Transm. Dis. 35, 269-275 (2008).

67. K. L. Vincent et al., "High resolution imaging of epithelial injury in the sheep cervicovaginal tract: a promising model for testing safety of candidate microbicides," Sex. Transm. Dis. 36, 312-318 (2009).

68. K. L. Vincent et al., "Monitoring vaginal epithelial thickness changes noninvasively in sheep using optical coherence tomography," Am. J. Obstet. Gynecol. 208(4), 282.e1-282.e7 (2013).

69. T. Kalganova et al., "The use of cross-polarization OCT in determining the dynamics of the state of pathological and normal tissues during radiation and photodynamic therapy," Mod. Technol. Med. 7, 119-129 (2015).

70. C. Radecki-Breitkopf et al., "Acceptability of optical coherence tomography and abstinence requirements among women participating in microbicide safety trials," Sex. Transm. Dis. 39, 28-31 (2012).

71. M. Ascencio et al., "The role and value of optical coherence tomography in gynecology," J. Gynecol. Obstet. Biol. Reprod. 36, 749-755 (2007).

72. N. Shakhova, F. Feldchtein, and A. Sergeev, "Applications of optical coherence tomography in gynecology," in Handbook of Optical Coherence Tomography, B. E. Bouma and Tearney G. E., Eds., pp. 649-672, Marcel Dekker, Inc., Basel, New York (2002).

73. V. V. Tuchin, R. K. Wang, and A. T. Yeh, "Optical clearing of tissues and cells," J. Biomed. Opt. 13, 021101 (2008).

74. C. Banzhaf et al., "Optical coherence tomography imaging of nonmelanoma skin cancer undergoing imiquimod therapy," Skin Res Technol. 20, 170-176 (2014).
75. M. Mogensen et al., "Optical coherence tomography for imaging of skin and skin diseases," Semin. Cutaneous Med. Surg. 28(3), 196-202 (2009).

76. M.-T. Tsai et al., "Monitoring of wound healing process of human skin after fractional laser treatments with optical coherence tomography," Biomed. Opt. Express 4, 2362-2375 (2013).

77. U. Mahmood et al., "In vivo optical coherence tomography of the nasal mucosa," Am. J. Rhinol. 20, 155-159 (2006).

78. R. Allison et al., "PD/PDT for gynecological disease: a clinical review," Photodiagn. Photodyn. Ther. 2, 51-63 (2005).

79. N. Shishkova, O. Kuznetsova, and T. Berezov, "Photodynamic therapy for gynecological diseases and breast cancer," Cancer Biol. Med. 9, 9-17 (2012).

80. Y.-K. Park and C.-H. Park, "Clinical efficacy of photodynamic therapy," Obstet. Gynecol. Sci. 59, 479-488 (2016).

Mikhail Kirillin is a senior researcher at the Laboratory of Biophotonics at the Institute of Applied Physics at the Russian Academy of Sciences. He received his PhD from Moscow State University, Russia in 2006 and DrSc (Tech.) degree from the University of Oulu, Finland in 2008. His scientific interests include biomedical imaging modalities, theoretical description and numerical simulations of light transport in biotissues and other scattering media (in particular, Monte Carlo technique), and photodynamic therapy.

Tatiana Motovilova received her PhD from Nizhny Novgorod State Medical Academy in 2005 in the area of obstetrics and gynecology. Currently, she is an associate professor at Nizhny Novgorod State Medical Academy and a practitioner at the Nizhny Novgorod City Clinical Hospital. Her scientific interests include the pathology of endometrium and cervix, fertility restoration, bioimaging, and laser medicine.

Natalia Shakhova is a leading researcher at the Laboratory of Biophotonics at the Institute of Applied Physics at the Russian Academy of Sciences. She received her PhD and DSc degrees from Nizhny Novgorod State Medical Academy in 1996 and 2005, respectively. She is an expert in clinical oncology, translation medicine, and photodynamic therapy. She has wide experience in application of optical imaging, therapeutic and surgical systems in clinical environment. 